\title{
Protective effect of chlorogenic acid on renal ischemia/reperfusion injury in rats
}

\author{
Tuncay Toprak ${ }^{1}$, Cagri Akin Sekerci ${ }^{2}$, Hasan Riza Aydın ${ }^{3}$, Mehmet Akif Ramazanoglu ${ }^{4}$, \\ Fatma Demet Arslan ${ }^{5}$, Banu Isbilen Basok ${ }^{5}$, Hatice Kucuk ${ }^{6}$, Huseyin Kocakgol ${ }^{3}$, Hamit Zafer Aksoy ${ }^{3}$, \\ Seyhan Sumeyra Asci ${ }^{7}$, Yılören Tanıdır ${ }^{8}$
}

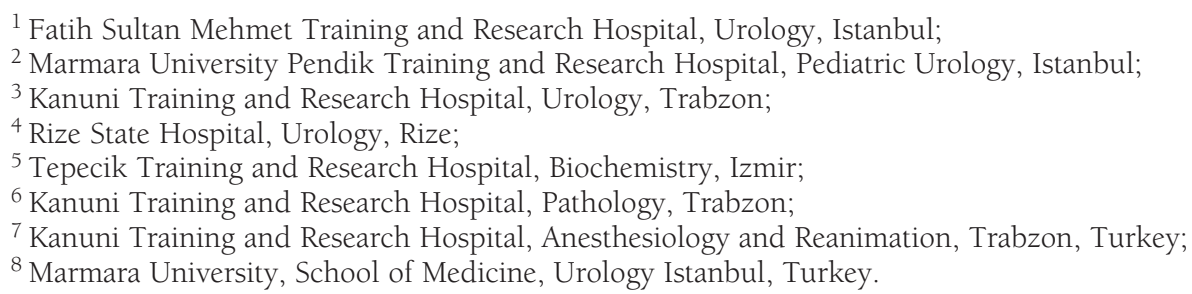

\begin{abstract}
Summary Objectives: Ischemia/reperfusion (I/R) injury is a common cause of renal injury and to date, many pharmacological agents have been identified to decrease I/R injury. One of the potential compound that can target $I / R$ injury is chlorogenic acid (CGA). It has potent antiinflammatory, antibacterial, anti-oxidant, analgesic and antipyretic activities in in vitro experiments and in vivo animal models. The aim of the study was to investigate the protective characteristic of CGA on renal I/R injury.
\end{abstract}

Material and Methods: 24 rats were randomly allocated to three groups $(n=8)$ : Sham, I/R+CGA and I/R groups. CGA was administered intraperitoneally at a dose of $20 \mathrm{mg} / \mathrm{kg}, 10$ min before reperfusion. I/R injury was achieved by clamping the left renal artery for 45 minutes, followed by reperfusion for 4 hours. The left kidneys of the rats were examined for tissue damage by histopathological and biochemical examination. For histological evaluation, EGTI scoring system was used. For biochemical examination total oxidant status, total antioxidant status and oxidative stress index were used. The power analysis indicated that 8 subjects per group would be required to produce $80 \%$ chance of achieving statistical significance at $p<0.05$ level. The results are expressed as mean \pm SD. MannWhitney $U$ was performed for statistical analysis. Results: Histopathological examination of the tissue damage revealed that all kidneys in the sham group were normal. I-R group had significantly higher histopathological scores than other groups. Histopathological improvement was seen after CGA treatment. TAS, TOS and OSI values of I-R group were significantly higher than sham group (0.88 vs 0.76 ( $p: 0.004)$, 13.8 vs 7.04 (p: 0.021) and 0.15 vs 0.09 (p: 0.034), respectively). In CGA treated group TAS, TOS and OSI levels were $0.84,6.47$ and 0.07 , respectively. CGA treatment resulted in significant improvement in TOS and OSI parameters. Conclusions: CGA treatment provided marked improvement in renal histology and suppressed oxidative stress. Thus, CGA may have a protective effect in renal tissue against I/R injury.

KEY WORDS: Renal ischemia; Oxidative stress; Chlorogenic acid; Rat.

Submitted 29 November 2019; Accepted 12 December 2019

\section{INTRODUCTION}

Ischemia/reperfusion (I/R) injury is a common cause of renal injury arising from a variety of clinical circumstances, including partial nephrectomy, renal transplan- tation, iatrogenic trauma, sepsis and shock $(1,2)$. It is characterized by restriction of the blood flow, followed by restoring the blood flow and oxygenation. Cessation of blood flow causes ischemia and tissue damage. Restoration of blood flow during reperfusion period, often leads to exacerbation of these harmful events instead of improving (3). The pathologic processes underlying this injury are complex and include interactions between the endothelium, cell death programs and immune system (4). Reactive oxygen radicals (ROR), necrosis, apoptosis, and inflammation plays a role in this process but the exact mechanisms remain unclear $(5,6)$. The production of ROR is considered a key reason for oxidative stress during the reperfusion period (6). Thus, targeting oxidative stress' processes is an ideal therapeutic approach. I/R injury in renal transplantation often leads to allograft dysfunction and increased rejection (7). In partial nephrectomy, renal tissue damage may occur due to clamping of renal arteries (8). In the clinical scenario, renal I/R models generated in animals are important to understand the pathophysiology of renal injury and the potential treatment options. To date, many pharmacological agents such as N-acetylcysteine (9), Allopurinol (10) or Mannitol (11) have been identified to decrease I/R injury after nephron sparing surgery. To prevent the kidney damage due to I/R injury, several anti-inflammatories and antioxidants have been used in experimental studies (12-14). Another potential compound that can target I/R injury is chlorogenic acid (CGA). It is formed by esterification of quinic and caffeic acids and it is one of the polyphenols abundant in the human diet (15). It has potent anti-inflammatory, antibacterial, anti-oxidant, analgesic and antipyretic activities in in vitro experiments and in vivo animal models (16-18). Thus, we investigate protective effects of CGA against renal I/R injury in an in vivo rat model which may potentially help us in urological surgeries such as partial nephrectomy and renal transplantation where clamping is required. To examine this, we evaluated histopathological findings and biochemical analyses (including

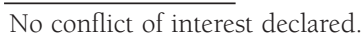


total oxidant (TOS) status, total antioxidant (TAS) assays). In our knowledge, there have been no studies concerning the protective effect of CGA against renal I/R injury.

\section{Materials AND MEthods}

The experimental and surgical procedures were conducted according to routine animal care guidelines, and the Guide for the Care and Use of Laboratory Animals (19).

The approval was obtained from Institutional Animal Care and Use Committee of Karadeniz Technical University (Trabzon, Turkey) (Approval Number/ID: 2019/5). 24 male Sprague-Dawley rats (8 weeks old, weight 230-300 g) were purchased from the Karadeniz Technical University Laboratory Animals Research Centre (Trabzon, Turkey).

All animals were kept in captivity under the same nutritional and environmental conditions. Rats were entrained under a 12:12 h dark: light cycle (lights on 6 am- 6 pm) with stable temperature $\left(21 \pm 2^{\circ} \mathrm{C}\right)$ and humidity $(60 \pm 5 \%)$.

The rats had sterile water and food available ad libitum.

\section{Experimental design}

Rats were randomly and equally divided into 3 groups;

1. Vehicle-treated ischemic (I/R): After sterile conditions were obtained, a midline laparotomy was performed. Isotonic saline (1 mg/kg) was applied intraperitoneally $10 \mathrm{~min}$ before the beginning of reperfusion. The left kidney pedicle was clamped with an artery clamp for 45 minutes. After 45 minutes of left renal ischemia, the occlusion clamp was removed for reperfusion for 4 hours and the incision was closed.

2. Vehicle-treated sham (Sham):

Rats underwent the same surgical procedures except unilateral renal occlusion. During the experiment, they were kept under anesthesia with gauze, soaked in saline in the abdominal cavities.

3. CGA-treated ischemic $(I / R+C G A)$ : After sterile conditions were obtained, a midline laparotomy was performed. CGA (Sigma-Aldrich) $(20 \mathrm{mg} / \mathrm{kg}$ ) was applied intraperitoneally $10 \mathrm{~min}$ before the beginning of reperfusion. The left kidney pedicle was clamped with an artery clamp for 45 minutes. After 45 minutes of left renal ischemia, the occlusion clamp was removed and the incision was closed.

\section{Administration of CGA}

CGA was dissolved in saline (vehicle) and administered intraperitoneally at a total dose of $20 \mathrm{mg} / \mathrm{kg} 10$ minutes before reperfusion.

\section{Surgical procedure}

For anesthetic ketamine hydrochloride $(100 \mathrm{mg} / \mathrm{kg}$, Ketalar, Eczacıbasi, Turkey) and xylazine (10 mg/kg) were used intraperitoneally. Following fluid replacement with $3 \mathrm{~mL} \cdot \mathrm{kg}-1 \cdot \mathrm{h}-1$ lactated Ringer's solution, the surgical area was prepared for sterilization. Then a midline laparotomy incision was performed and the left kidney pedicle was dissected. Left renal ischemia was induced by clamping the left renal artery for $45 \mathrm{~min}$ for the I/R and I/R-CGA groups. For reperfusion the clamp was removed and the pulsation of renal artery was verified visually. After controlling the bleeding, the skin layers were sutured. The rats were sacrificed $4 \mathrm{~h}$ after completion of the reperfusion and the left kidneys were removed and stored for biochemical and histopathological examination under favorable conditions.

\section{Histological analysis}

Removed kidney was fixed with 10\% formalin and embedded in paraffin. $5 \mu \mathrm{m}$ tissue sections obtained for Hematoxylin and Eosin staining. An experienced, independent pathologist, who was blinded to the groups, analyzed three different tissue sections in each group, using a Zeiss Axio Imager A2 microscope (Carl Zeiss AG, Germany). The histological evaluations of the renal tissue were graded as described in the study of Medeiros et al. (20) (Table 1). The scores were applied to microscopic changes consistent with tubular necrosis: vacuolization of tubular cells, tubular lumen dilation, intra-tubular cylinders, interstitial fibrosis and tubular cell necrosis. For histological evaluation, EGTI scoring system, which was developed especially for animal studies in kidney tissues in the context of injury, was also used (21), (Table 2). This system consists of histological damage in 4 separate components: Endothelial, Glomerular, Tubular, and Interstitial.

\section{TAS and TOS assays}

The serum TAS and TOS levels were determined with a

\section{Table 1.}

Scoring system for renal histopathology.

\begin{tabular}{|l|c|}
\hline Score & Histopathological pattern \\
\hline 0 & Normal \\
\hline 0.5 & Small focal damaged areas \\
\hline 1 & $<10 \%$ Cortical damaged zone \\
\hline 2 & $10-25 \%$ Cortical damaged zone \\
\hline 3 & $25-75 \%$ Cortical damaged zone \\
\hline 4 & $>75 \%$ Cortical damaged zone \\
\hline
\end{tabular}

Table 2.

The EGTI histology scoring system.

\begin{tabular}{|c|c|c|}
\hline Tissue type & Damage & Score \\
\hline \multirow[t]{5}{*}{ Tubular } & No damage & 0 \\
\hline & $\begin{array}{l}\text { Loss of Brush Border (BB) in less than } 25 \% \text { of tubular cells. } \\
\text { Integrity of basal membrane }\end{array}$ & 1 \\
\hline & $\begin{array}{l}\text { Loss of BB in more than } 25 \% \text { of tubular cells, Thickened basal } \\
\text { membrane }\end{array}$ & 2 \\
\hline & $\begin{array}{l}\text { (Plus) Inflammation, cast formation, necrosis up to } 60 \% \\
\text { of tubular cells }\end{array}$ & 3 \\
\hline & (Plus) Necrosis in more than $60 \%$ of tubular cells & 4 \\
\hline \multirow[t]{4}{*}{ Endothelial } & No damage & 0 \\
\hline & Endothelial swelling & 1 \\
\hline & Endothelial disruption & 2 \\
\hline & Endothelial loss & 3 \\
\hline \multirow[t]{4}{*}{ Glomerular } & No damage & 0 \\
\hline & Thickening of Bowman capsule & 1 \\
\hline & Retraction of glomerular tuft & 2 \\
\hline & Glomerular fibrosis & 3 \\
\hline \multirow[t]{5}{*}{ Tubulo/Interstitial } & No damage & 0 \\
\hline & Inflammation, haemorrhage in less than $25 \%$ of tissue & 1 \\
\hline & (Plus) necrosis in less than $25 \%$ of tissue & 2 \\
\hline & Necrosis up to $60 \%$ & 3 \\
\hline & Necrosis more than $60 \%$ & 4 \\
\hline
\end{tabular}


novel automatic method, developed by Erel (22, 23). The ratio of TAS to TOS is defined as oxidative stress index (OSI), expressed as percentage.

\section{Statistical analysis}

IBM SPSS 22 version (SPSS IBM, Turkey) program was used for analysis. Before starting to study, we performed power analysis. The power analysis indicated that 8 subjects per group would be required to produce $80 \%$ chance of achieving statistical significance at $p<0.05$ level. The Kolmogorov-Smirnov test was performed to determine the normality of data. The results are expressed as mean $\pm \mathrm{SD}$. Mann-Whitney $\mathrm{U}$ was performed for statistical analysis, as appropriate. A p value below 0.05 was considered statistically significant.

\section{Table 3.}

Histopathology scoring of cortical damage of the groups.

\begin{tabular}{cccc|}
\hline Rats & Sham group & I/R group & I/R + CGA group \\
\hline 1 & 0 & 0.5 & 0.5 \\
\hline 2 & 0 & 1 & 0 \\
\hline 3 & 0 & 1 & 1 \\
\hline 4 & 0 & 1 & 0.5 \\
\hline 5 & 0 & 0.5 & 0.5 \\
\hline 6 & 0 & 0.5 & 0.5 \\
\hline 7 & 0 & 1 & 1 \\
\hline 8 & 0 & 0.5 & 0.5 \\
\hline
\end{tabular}

Table 4.

Comparison of rats in terms of EGTI scoring.

\begin{tabular}{cccc|}
\hline Rats & Sham group & I/R group & I/R + CGA group \\
\hline 1 & 0 & 8 & 5 \\
\hline 2 & 0 & 7 & 3 \\
\hline 3 & 0 & 8 & 6 \\
\hline 4 & 0 & 8 & 3 \\
\hline 5 & 0 & 5 & 3 \\
\hline 6 & 0 & 5 & 4 \\
\hline 7 & 0 & 8 & 7 \\
\hline 8 & 0 & 4 & 6
\end{tabular}

Figure 1.

Histological images of rat renal cortex sections. a; Normal renal cortex (sham group), b; tubular necrosis $(\mathrm{I} / \mathrm{R}), \mathrm{c}$; tubular injury (I/R+CGA group.

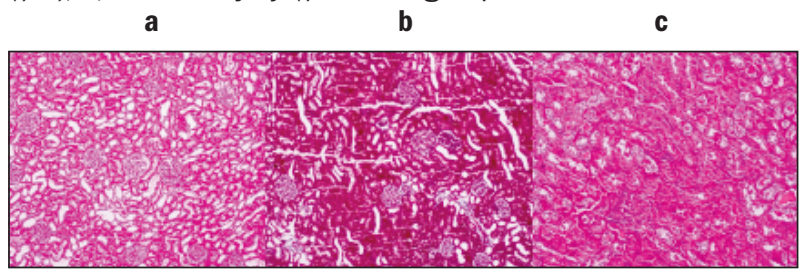

\section{RESULTS}

CGA showed histopathologic improvement in ischemia reperfusion injury as shown in Tables 3 and 4 . All rats in the sham group had normal histopathological findings. By contrast, as shown in table 3, 4 (50\%) rats in the I/R group had small focal damaged areas and 4 (50\%) had $<10 \%$ cortical damage. In I/R+CGA group, 1 (12.5\%) rat had normal kidney, 5 (62.5\%) had small focal damaged areas and 2 (25\%) had $<10 \%$ cortical damage. EGTI scores of the rats in each group are shown in Table 4, separately. The pathological figures were shown in Figure 1. As shown in Table 5, CGA improved biochemical values. TAS, TOS and OSI values of the sham group was significantly lower than I/R group (P: 0.004, 0.021, 0.034 , respectively). There was no significant difference between the sham and I/R + CGA groups in terms of TOS and OSI values (P: $0.83,0.52$, respectively). TOS and OSI values of the I/R group were significantly higher than the other groups ( $\mathrm{P}: 0.021,0.034$, respectively for comparison of sham and I/R groups and P: 0.046, 0.040, respectively for comparison of I/R+CGA and I/R groups).

\section{Discussion}

Renal I/R injury is a major reason for renal dysfunction. It induces an inflammatory response and oxidative stress. At the site of inflammation, leukocytes infiltration occurs and results in the secretion of pro-inflammatory cytokines, including TNF- $\alpha$, HMGB1, IL-6, and IL-1 $\beta$ (24). ROR, produced during reperfusion is considered to play a central role in I/R injury by direct attack on multiple molecule sequences. In living organisms, ROR arise as a result of normal biological metabolism and they can distort the structures of DNA, fats, proteins and carbohydrates. To ensure I/R experimentally, the left renal artery was occluded for $45 \mathrm{~min}$. It was shown that the 45 min model of IR injury used here provides reproducible and robust assessment of treatment effects against IR injury $(25,26)$. Oxidative stress and antioxidant status can be assessed by several markers and various methods. However, it is both time-consuming and costly to measure these markers separately (27). For this reason, in this study we used TOS, TAS and OSI levels to measure the oxidative stress status. In recent years it has become more common to measure these values $(23,28,29)$. In this study TAS, TOS and OSI levels were found to be significantly higher in I/R group compared to sham group and CGA alleviated these parameters. The histopathological classification system presented in Table 1 was used for histological diagnosis. However, since this system shows only cortical damage. Renal IR injury is a complex process which effects the glomerular, tubulo-interstitial and endothelial cells. Acute tubular necrosis, loss of endothelial cell integrity, glomerular

\section{Table 5.}

Comparison of groups in terms of biochemical parameters.

\begin{tabular}{|lcccccc|}
\hline & TAS median (min-max) & $\mathbf{P}$ & TOS median (min-max) & $\mathbf{P}$ & 0SI median (min-max) & $\mathbf{P}$ \\
\hline Group 1-2 & $0.84(0.76-1)-0.76(0.66-0.80)$ & 0.021 & $6.47(2.1-23.7)-7.04(4.7-13.9)$ & 0.83 & $0.07(0.03-0.26)-0.09(0.06-0.21)$ & 0.52 \\
\hline Group 2-3 & $0.76(0.66-0.80)-0.88(0.75-0.98)$ & 0.0048 & $7.04(4.7-13.9)-13.8(6.4-18.5)$ & 0.021 & $0.09(0.06-0.21)-0.15(0.09-0.19)$ & 0.034 \\
\hline Group 1-3 & $0.84(0.76-1)-0.88(0.75-0.98)$ & 0.49 & $6.47(2.1-23.7)-13.8(6.4-18.5)$ & 0.046 & $0.07(0.03-0.26)-0.15(0.09-0.19)$ & 0.040 \\
\hline (1) I-R +CGA, (2) sham, (3) I-R. Mann Whitney U test & & & & & \\
\hline
\end{tabular}


ischemic damage and tubulo-interstitial damage are the hallmarks of renal IR injury which is important for complete and comprehensive documentation. For this reason, EGTI scoring system was used together with other system. Because it is reliable, simple, more informative and more detailed scoring system about the degree of tissue damage of the kidney (21). The histological study showed tubular dilation, tubular necrosis, cellular edema and inflammatory cell infiltration in the tubular interstitium. These lesions were less intense in CGA treated rats compared to untreated animals.

In order to block inflammatory response and oxidative stress, several drugs have been used to prevent renal I/R injury in several experimental studies $(13,14)$. However, the new experimental studies will help us to find the most appropriate feasible treatment. In the present study, CGA was examined for its potential effects on regulating renal I/R injury. CGA is a polyphenol, which is abundantly found in coffee, fruits and vegetables. It has been used as an antioxidant, analgesic and anti-inflammatory. It has a certain number of $\mathrm{R}-\mathrm{OH}$ radicals that are capable of forming the hydrogen free radical, thereby protecting tissue cells from oxidative damage (30). It has been shown to act as a scavenger of hydroxyl radicals, peroxynitrite and superoxide radicals in a concentrationdependent manner in vitro (31). In the study of Yun et al. (32) CGA given at $10 \mathrm{mg} / \mathrm{kg}$ intraperitoneally, $10 \mathrm{~min}$ before ischemia and reperfusion was chosen as the most effective dose for histology evaluation for I/R- induced hepatic injury.

In our study, it was administered intraperitoneally at a total dose of $20 \mathrm{mg} / \mathrm{kg} 10$ minutes before reperfusion. Previous studies in rat models have shown that CGA is protective against hepatic and focal cerebral I/R injury $(32,33)$. We have observed that CGA has a protective effect against renal I/R injury in our study. We considered that CGA may serve a protective role in the rat model of renal I/R injury. To the best of our knowledge, there is no data showing the effect of CGA on I/R kidney injury and evaluating TAS, TOS levels and histopathology together.

As a limitation of our study, since we did not perform a right nephrectomy, we did not measure plasma creatinine, the most commonly used marker as a measure of renal excretory function (34).

\section{Conclusions}

CGA treatment provided marked improvement in renal histology and suppressed oxidative stress. Thus, CGA may have a protective effect in renal tissue against I/R injury.

\section{References}

1. Sagiroglu T, et al. Effects of apelin and leptin on renal functions following renal ischemia/reperfusion: An experimental study. Exp Ther Med. 2012; 3: 08-914.

2. Snoeijs MG, et al. Acute ischemic injury to the renal microvasculature in human kidney transplantation. Am J Physiol Renal Physiol. 2010; 299:F1134-40.

3. Orvieto MA, et al. Ischemia preconditioning does not confer resilience to warm ischemia in a solitary porcine kidney model. Urology. 2007; 69:984-987.

4. Eltzschig HK. Eckle T Ischemia and reperfusion-from mechanism to translation. Nat Med. 2011; 17:1391.

5. Zhang J, et al. Erythropoietin pretreatment ameliorates renal ischaemia-reperfusion injury by activating PI3K/Akt signalling. Nephrology (Carlton). 2015; 20:266-72.

6. Wang $L$, et al. Effect of picroside II on apoptosis induced by renal ischemia/reperfusion injury in rats. Exp Ther Med. 2015; 9:817-822.

7. Fadili W, Allah MH, Laouad I. Chronic renal allograft dysfunction: risk factors, immunology and prevention. Arab J Nephrol Transplant. 2013; 6:45-50.

8. Martin GL, et al. Comparison of total, selective, and nonarterial clamping techniques during laparoscopic and robot-assisted partial nephrectomy. J Endourol. 2012; 26:152-156.

9. Conesa EL, et al. N-acetyl-L-cysteine improves renal medullary hypoperfusion in acute renal failure. Am J Physiol Regul Integr Comp Physiol, 2001; 281: R730-7.

10. Rhoden E et al., Protective effect of allopurinol in the renal ischemia--reperfusion in uninephrectomized rats. Gen Pharmacol. 2000; 35:189-93.

11. Feitoza CQ, et al. Cyclooxygenase 1 and/or 2 blockade ameliorates the renal tissue damage triggered by ischemia and reperfusion injury. Int Immunopharmacol. 2005; 5:79-84.

12. Sahna E, et al. The protective effects of physiological and pharmacological concentrations of melatonin on renal ischemia-reperfusion injury in rats. Urol Res. 2003; 31:188-193.

13. Hosseini F, et al. Effect of beta carotene on lipid peroxidation and antioxidant status following renal ischemia/reperfusion injury in rat. Scand J Clin Lab Invest. 2010; 70:259-263.

14. Kizilgun $M$, et al. Beneficial effects of N-acetylcysteine and ebselen on renal ischemia/reperfusion injury. Ren Fail. 2011; 33:512-517.

15. Suzuki A, et al. Chlorogenic acid attenuates hypertension and improves endothelial function in spontaneously hypertensive rats. J Hypertens. 2006; 24:1065-1073.

16. Dos Santos MD, et al. Evaluation of the anti-inflammatory, analgesic and antipyretic activities of the natural polyphenol chlorogenic acid. Biol Pharm Bull. 2006; 29:2236-2240.

17. Almeida AAP, et al. Antibacterial activity of coffee extracts and selected coffee chemical compounds against enterobacteria. J Agricol Food Chem. 2006; 54:8738-8743.

18. Kono $Y$, et al. Iron chelation by chlorogenic acid as a natural antioxidant. Biosci Biotechnol Biochem. 1998; 62:22-27.

19. Council NR. Guide for the Care and Use of Laboratory Animals. 1996, Washington, DC: The National Academies Press. 140.

20. Medeiros PJD, et al. Effect of sildenafil in renal ischemia/reperfusion injury in rats. Acta Cir Bras. 2010; 25:490-495.

21. Chavez R, et al. Kidney ischaemia reperfusion injury in the rat: the EGTI scoring system as a valid and reliable tool for histological assessment. Journal of Histology and Histopathology. 2016; 3.

22. Erel O. A novel automated method to measure total antioxidant response against potent free radical reactions. Clin Biochem. 2004; 37:112-119.

23. Erel O. A new automated colorimetric method for measuring total oxidant status. Clin Biochem. 2005; 38:1103-1111.

24. Ysebaert DK, et al. Identification and kinetics of leukocytes after 
severe ischaemia/reperfusion renal injury. Nephrol Dial Transplant. 2000; 15:1562-74.

25. Delbridge $M$, et al. The effect of body temperature in a rat model of renal ischemia-reperfusion injury. in Transplantation proceedings. 2007. Elsevier.

26. Wystrychowski W, et al. Nephroprotective Effect of Pentoxifylline in Renal Ischemia-Reperfusion in Rat Depends on the Timing of Its Administration. in Transplantation proceedings. 2014. Elsevier.

27. Tarpey MM, Wink DA, Grisham MB. Methods for detection of reactive metabolites of oxygen and nitrogen: in vitro and in vivo considerations. Am J Physiol Regul Integr Comp Physiol. 2004; 286:R431R444.

28. Erel O. A novel automated direct measurement method for total antioxidant capacity using a new generation, more stable ABTS radical cation. Clin Biochem. 2004; 37:277-285.

29. Harma M, Erel O. Increased oxidative stress in patients with hydatidiform mole. Swiss Med Wkly. 2003; 133:563-6.

\section{Correspondence}

Tuncay Toprak, MD (Corresponding Author)

drtuncay55@hotmail.com

Fatih Sultan Mehmet Training and Research Hospital, Urology, Istanbul,

Turkey

E5 karayolu üzeri Fatih Sultan Mehmet Hastanesi C Blok Kat 3 icerenkoy

Atasehir/Istanbul

Çagn Akın Sekerci, MD

cagri_sekerci@hotmail.com

Marmara University Pendik Training and Research Hospital, Pediatric Urology,

Istanbul, Turkey

Hasan Riza Aydin, Assoc. Prof.

hrizaaydin@gmail.com

Huseyin Kocakgol, MD

hsynkocakgl@gmail.com

Hamit Zafer Aksoy, Ass. Prof.

hamitzaferaksoy@hotmail.com

Kanuni Training and Research Hospital, Urology, Trabzon, Turkey

Mehmet Akif Ramazanoglu, MD

maramazanoglu@hotmail.com

Rize State Hospital, Urology, Rize, Turkey

Fatma Demet Arslan, Assoc. Prof.

fatmademet.arslan@gmail.com

Tepecik Training and Research Hospital, Biochemistry, Izmir, Turkey

Banu Isbilen Basok, Assoc. Prof.

drisbilen@yahoo.com

Tepecik Training and Research Hospital, Biochemistry, Izmir, Turkey

Hatice Kucuk, Ass. Prof.

dr.hatice.kucuk@hotmail.com

Kanuni Training and Research Hospital, Pathology, Trabzon, Turkey

Seyhan Sumeyra Asci, MD

seyransumeyra@hotmail.com

Kanuni Training and Research Hospital, Anesthesiology and Reanimation,

Trabzon, Turkey

Yllören Tanıdır, Assoc. Prof.

yiloren@yahoo.com

Marmara University, School of Medicine, Urology Istanbul, Turkey
30. Zhang J, et al. Liquid chromatograph/tandem mass spectrometry assay for the simultaneous determination of chlorogenic acid and cinnamic acid in plasma and its application to a pharmacokinetic study. J Pharm Biomed Anal .2010; 51:685-690.

31. Graziani $G$, et al. Apple polyphenol extracts prevent damage to human gastric epithelial cells in vitro and to rat gastric mucosa in vivo. Gut. 2005; 54:193-200.

32. Yun N, Kang J-W, Lee S-M. Protective effects of chlorogenic acid against ischemia/reperfusion injury in rat liver: molecular evidence of its antioxidant and anti-inflammatory properties. J Nutr Biochem. 2012; 23:1249-1255.

33. Miao M, et al. Protective effect of chlorogenic acid on the focal cerebral ischemia reperfusion rat models. Saudi Pharm J. 2017; 25:556-563.

34. Suzuki $Y$, et al. Clinical validity of renal function markers including serum cystatin $C$ on chronic kidney disease classification. Rinsho Byori. 2011; 59:345-351. 